\title{
Cytological Studies on the Life-cycle in Absidia glauca Hagem (Mucorales)
}

\author{
Rolf Duus and Morten M. Laane \\ Institute of General Genetics, P. B. 1031, University of Oslo, \\ Blindern, Oslo 3, Norway
}

Received February 28, 1983

The life-cycle and nuclear conditions in Zygomycetes present still problems in spite of more than 100 years research. The process of conjugation was discovered as early as 1820 by Ehrenberg. In two papers from 1984 Dangeard and Leger made important observations on nuclear behaviour in the zygospores. Blakeslee (1904, 1906) examined different species, with the aim of establishing them as experimental material and to develop a field of fungal genetics. Unfortunately, nuclear behaviour both during formation, dormancy and germination of the zygospores remained obscure. Different problems arose, involving absence of regular germination of the zygospores and not at least, problems of staining and studying the tiny nuclei. A number of partly conflicting papers appeared due to substantial interpretative problems. Of especial interest here are the two works of Cutter (1942a, b) both being based on very extensive studies. In some particulars they contradict the conclusions of Lendner (1908) and of Keene (1914) and to a larger degree substantiate those of Ling-Young (1930-1931). Cutter's papers are important as they discuss the possibility of different nuclear behaviour between various species. As late as 1982 Esser writes that the processes of nuclear fusion and meiosis which both take place in the zygospore are still only partly explored.

The existing genetic and cytological studies suggest that haploid nuclei fuse in pairs. During germination of the zygospore only one of the diploid nuclei divides meiotically, the rest degenerate. Thus, in principle, the numerous spores in the zygosporangium arise from the four meiotic products of the meiotic nucleus, showing a $1: 1$ ratio for both crossing alleles, However, deviations from this may occur (Eslava et al. 1975, Cerda-Olmeido 1975, Gauger 1977).

In a previous paper one of us (Laane 1974) examined parts of the life cycle in the species Absidia glauca Hagem. Later, development of improved and more sensitive techniques such as the Feulgen-fluorescence method (ref. Laane and Lie 1975) made us able to reveal more details about the life-cycle, which are presented here.

Abbreviations used:

(LM): Light microscopy, (DIK): Differential interference contrast microscopy (Nomarsky), (AO): Acridine orange, (OS): Orcein staining, (AOFL): Acridine orange fluorescence microscopy, (FFL): Feulgen fluorescence microscopy, (TEM): Transmission electron microscopy, (SEM): Scanning electron microscopy. 


\section{Materials and methods}

We used $A$. glauca strains BS $42370(+)$ and CBS $10148(-)$ obtained from Centzaalbureau voor Schimmelcultures, Baarn. The species was originally isolated by Hagem (1908) from soil material. The fungus was grown on a medium consisting of $15 \mathrm{~g}$ sucrose, $1 \mathrm{~g}$ yeast extract, $20 \mathrm{~g}$ agar and $10 \mathrm{~g}$ garden soil extract. Distilled water was added to $1000 \mathrm{ml}$ and $\mathrm{pH}$ adjusted to 6,9 . The medium was autoclaved at $120^{\circ} \mathrm{C}$ for $20 \mathrm{~min}$.

Petri dishes were inoculated with spores of each strain $5 \mathrm{~cm}$ apart. Hyphae from the developing mycelia touched after 5 days and numerous zygospores formed. Cover-glasses smeared with a thin film of agar medium were suitable for making cultures for photography.

The best nuclear preparations for (LM) were obtained by (OS). A piece of clean "Cellotape" was placed upon mycelial growth. Parts of hyphae, spores and zygospores adhered to the tape in a single layer and were stripped off. The tape was fixed for $2 \mathrm{~min}$ in 3 parts absolute ethanol: 1 part glacial acetic acid for $5 \mathrm{~min}$. It was then hydrolyzed in $6 \mathrm{~N} \mathrm{HCl}$ at $22^{\circ} \mathrm{C}$ for $4 \mathrm{~min}$, carefully rinsed in distilled water for $30 \mathrm{~s}$ and then stained $2 \mathrm{~min}$ in aceto-orcein. The tape was placed with the hyphae upwards on a slide and a cover-glass was added. The nuclei obtained an intense orange-red colour, the cytoplasm remained mostly unstained. The preparations were embedded in Eukitt.

Nuclei were also examined by (FFL), (Laane and Lie 1975). Samples were fixed for $2 \mathrm{~min}$ in acetic-ethanol $1: 3$, then hydrolyzed for $5 \min$ in $1 \mathrm{~N} \mathrm{HCl}$ at $60^{\circ} \mathrm{C}$. After hydrolysis the tape with adhering mycelial growth was stained $10 \mathrm{~min}$ in the Feulgen solution, then rinsed in $0,5 \% \mathrm{SO}_{2}$-water for $5 \mathrm{~min}$. We embedded the preparations after dehydration in ethanol in Eukitt. For observations we used an incident Zeiss fluorescence microcsope (HBO $100 \mathrm{~W}$ mercury arch lamp, interference green filter BP $546 \mathrm{~nm}$, dichroitic mirror FT $580 \mathrm{~nm}$ and absorbtion filter LP 590 $\mathrm{nm})$. The nuclei fluoresced orange-red.

For (AOFL) we dissolved (AO) $1: 1000$ in tap water, pH 7,2, and stained , living samples with a drop of this solution on a slide. We used exitation filter 450 and absorbtion filter $530 \mathrm{~nm}$. Samples for (TEM) were fixed in $2 \%$ glutaraldehyde in $0,1 \mathrm{M} \mathrm{Na}$-cacodylate buffer. The specimens were post-fixed in the same buffer, dehydrated in ethanol and finally embedded in Epon. Both thick sections for (LM) and ultra-thin sections (1000 $\AA$ ) for (TEM) were made. As zygospores are extremely difficult to fix, some samples were treated with water-free formic acid according to Olson (1974). This treatment is said to degenerate chitine-acetate, thus enabling better preservation of cytoplasmatic structures. We stained sections for (LM) in a dilute alkaline toluidine-blue solution for $20 \mathrm{~s}$. (TEM) sections were stained in uranyl acetate and lead-citrate, then coated with carbon in a vacuum evaporator and examined in a Siemens electron microscope. (SEM) preparations were fixed in $2 \% \mathrm{OsO}_{4}$, dehydrated in ethanol and critical-point dried with $\mathrm{CO}_{2}$. After mounting on specimen stubs, we coated the preparations with gold-palladium and examined them in a Jeol microscope. 

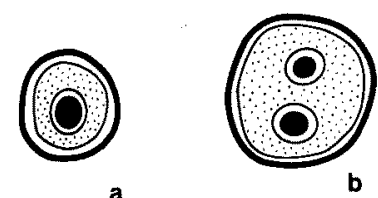

a
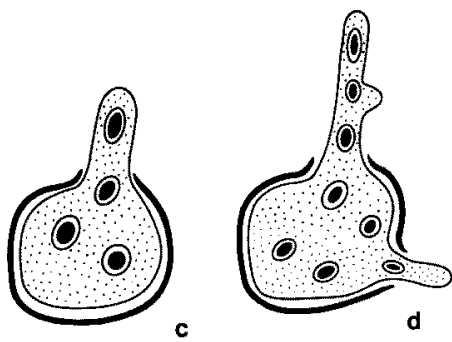
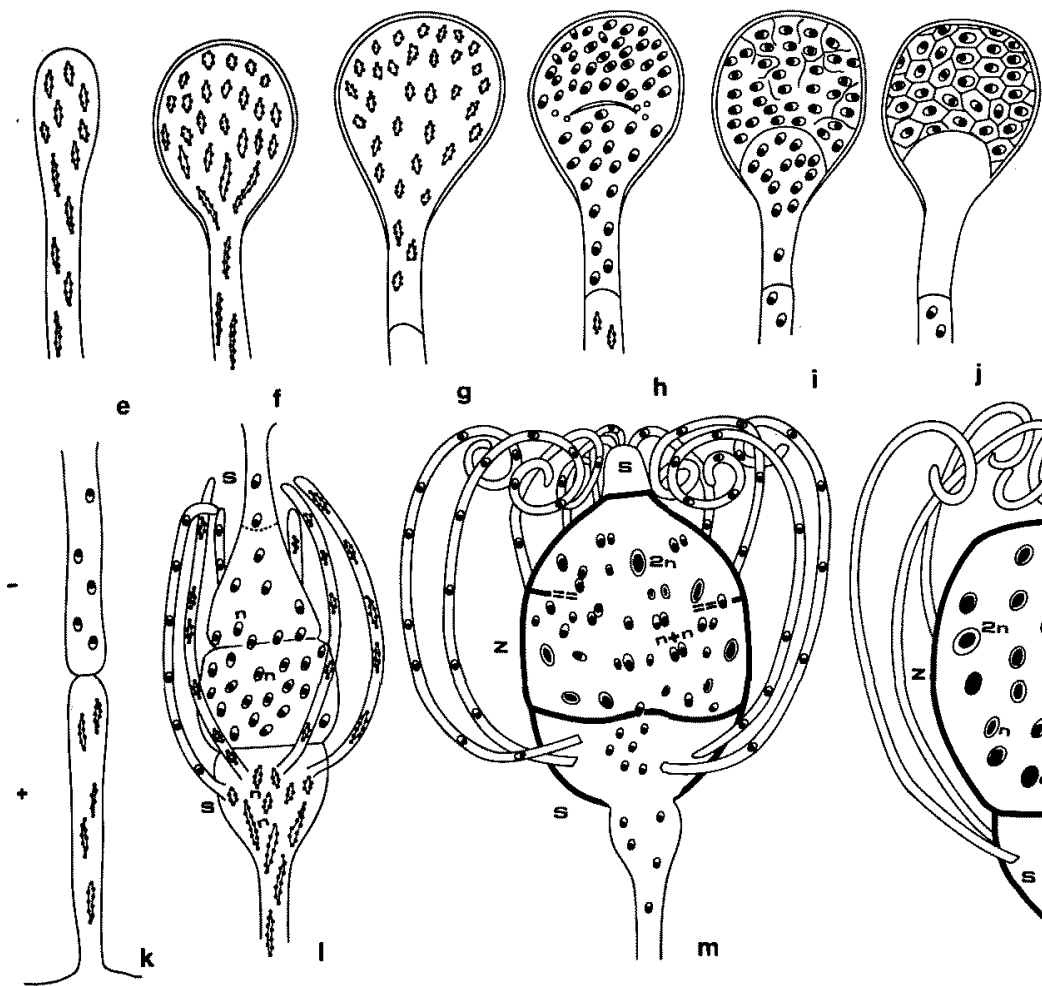

h

i

i
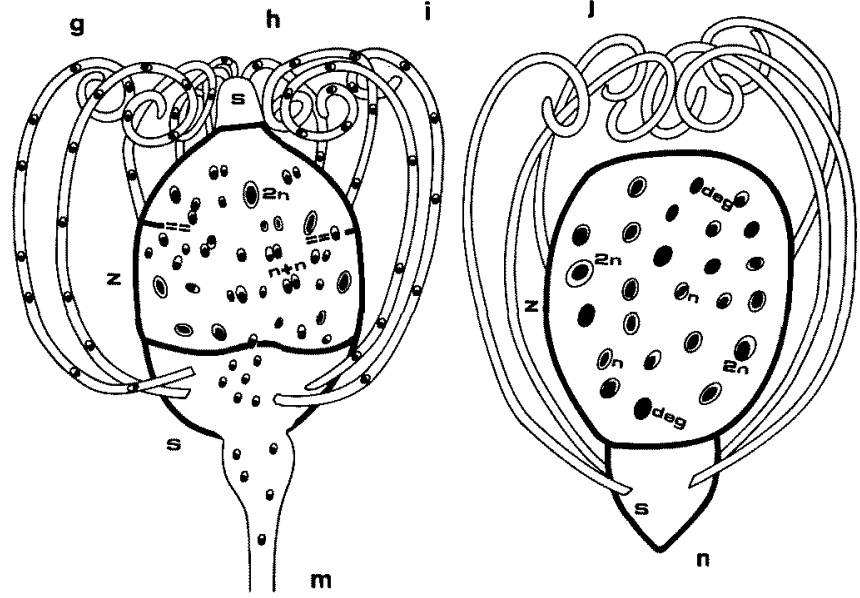

Fig. 1, a-n. Nuclear behaviour in important stages of life-cycle in Absidia glauca (compare with Fig. 13) as revealed by (OS) and (FFL). a-d, germination of vegetative spore. a, slightly swelled spore with single nucleus (time $2 \mathrm{~h}$ ). $\mathrm{b}$, later stage (time $4 \mathrm{~h}$ ) primary nuclear division completed. $\mathrm{c}-\mathrm{d}$, germination and nuclear migration into developing hyphae. e-j, development of sporangiospores. e, early stage with slight swelling of hyphal tip showing numerous nuclear divisions. $\mathrm{f}-\mathrm{g}$, later stages, numerous nuclei have migrated into developing sporangium, in $g$ the sporangium is cut off by a septum. h-j, later stages with development of columella. Nearly mature, single nucleated spores have formed in $j . \mathrm{k}-\mathrm{n}$. Development of zygospore. $\mathrm{k}$, early stage showing little difference between $(+)$ and $(-)$ hyphae. 1, later stage just before nuclear fusion, hyphal appendages developing from lower suspensor. $\mathrm{m}$, nuclear fusion occurs, the forming zygospore now contains a mixture of diploid and haploid nuclei. $\mathrm{n}$, later stage, some nuclei in process of degeneration, compare with Fig. $8, \mathrm{~m}$. $\mathrm{S}$ =suspensor cell, $\mathrm{Z}=$ zygospore. 
Results and discussion

\section{The vegetative nucleus}

The vegetative nucleus in $A$. glauca is spherical to slight ellipsoidal, about $1 \mu \mathrm{m}$ in diameter (Figs. $1 \mathrm{a}-\mathrm{d}, 8 \mathrm{a}-\mathrm{b}$ ). The vegetative mycelium is multinucleate (Fig. $5 \mathrm{a}-\mathrm{e}$ ) and nuclei are able to stream between various parts of the mycelia. The nuclear content has a slightly higher refraction index than the cytoplasm, appearing darker by phase contrast (positive mode). Each nucleus contains a nucleolus in its centre. Only sometimes the nucleolus is attached to the nuclear envelope. The nucleolus may show rapid Brownian movements in living nuclei and is the most refractive nuclear part. (TEM) shows the nuclear envelope to consist of two unit membranes $300 \AA$ apart.
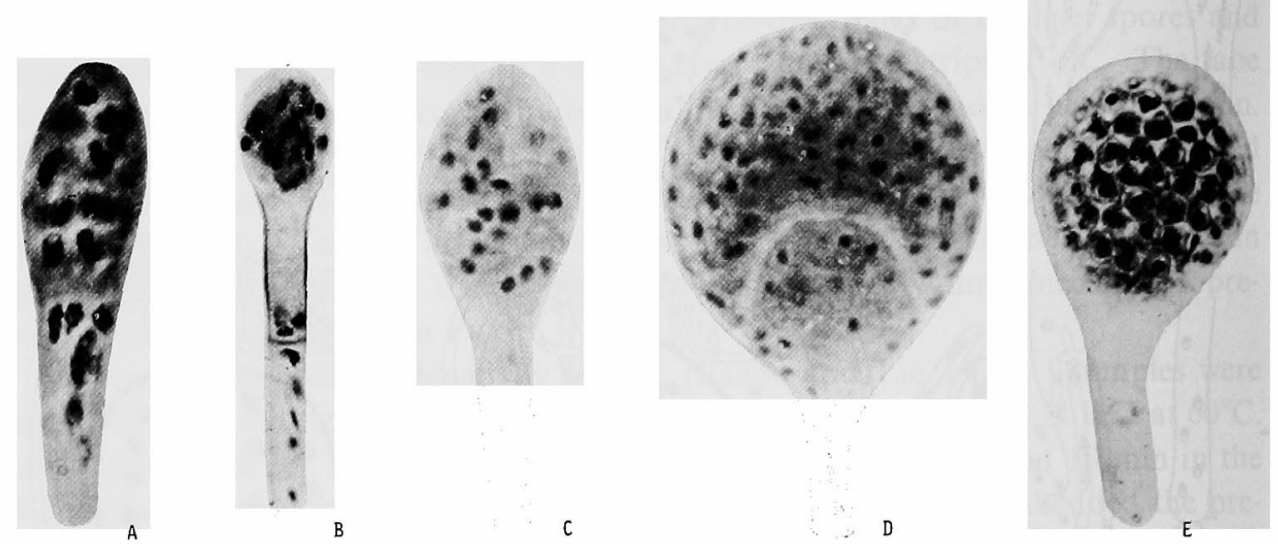

Fig. 2. (OS) of nuclear behaviour in developing vegetative sporangia of Absidia glauca. A, early stage of development, most nuclei are in division. B, sporangium cut off by a septum. C-E, further development. Note columella formed in $\mathrm{A}$ and single-nucleated spores in $\mathrm{E}$.

Our observations show that the nuclei differ from spherical or ellipsoidal shape during cytoplasmatic streaming and/or nuclear division (Fig. 1 e-g, Fig. 2 a). By (TEM) chromatin and chromosomes have the same electron density as the rest of the cytoplasm. Distinctly stained chromosomes are only possible to seen by (LM) with (AOFL), (OS) or (FFL). The nucleolus emits yellow-red light by (AOFL), (Fig. 11) and no light by (FFL). Nuclei believed to represent division stages show a number of chromatin containing "bodies". They emit green fluorescence light by (AOFL), orange-red fluorescence light by (FFL) (Fig. $5 \mathrm{~d}-\mathrm{e})$. The number of chromatin bodies varies in different nuclei. By classifying randomly selected nuclei having different numbers of chromatin bodies we obtained the diagram Fig. 6 which shows the nuclear population to contain two large groups, one with about 5 bodies and one with abour $10-11$ bodies. The first group represents slightly elongated nuclei, the latter more threadlike nuclei.

\section{Vegetative nuclear division}

Dividing nuclei may be arranged in characteristic groups according to the pattern of chromatin bodies: 
A) Slightly elongated nuclei with few identifiable bodies (5-6). They may be located peripherally, near the nuclear envelope. This stage corresponds to late prophase.

B) More elongated nuclei with bodies sometimes scattered or linearily arranged. Bodies of same size as in (A). This stage corresponds to metaphase, bodies in (A) and (B) to clustered chromosomes.

C) More elongated nuclei with apparently more or less linearily arranged bodies, 10-12 in a single "row" or a double "row". This stage corresponds to ana-

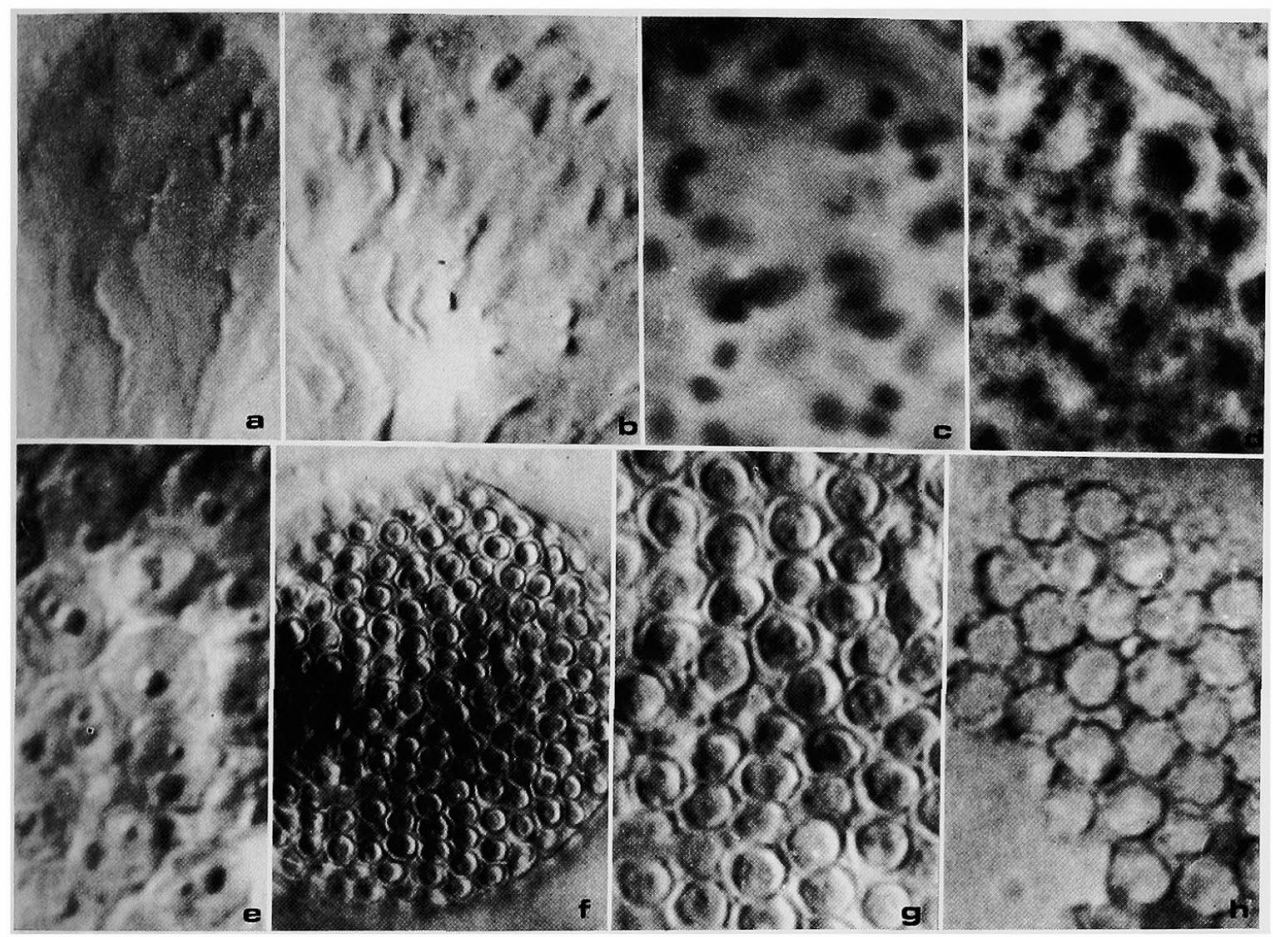

Fig. 3. Details of vegetative sporangium formation in Absidia glauca (a-g) and from zygospore (h). (a-b) and (e-g) (DIK), (c-d) (OS). a, early stage of nuclear migration into the sporangium. b, slightly later stage. c, nuclear division completed. d, compartmentalization of cytoplasm during spore delimitation. e, spore delimitation completed. f, sporangium with mature spores. $\mathrm{g}$, detail of f. h, honey-comb structure of cytoplasm in dormant zygospore (2 weeks old).

phase, bodies are mostly separating chromatids. Nuclei with scattered chromatids also occur.

We did not find the chromosomes arranged in metaphase plates in any of the several thousand nuclei examined. The chromatid separation is obviously asynchronous (Fig. 7). On basis of this and the previous study (Laane 1974) we interpret our observations in the following manner:

Each end in an elongated nucleus represents a polar area. In each a microtubule organizing center (MTOC) is situated in a constriction in the nuclear envelope. Rays of microtubules appear to pass from pole-to-pole. We were not able to observe microtubular attachment and to identify kinetochores. Kubai (1978) 
has extensively discussed the problems with the observed, socalled two-track or double-bar chromatin distribution in dividing nuclei which is common in many fungi. Day (1972) pointed out that about $70-80 \%$ of the basidiomycetes for which nuclear illustrations are available display the two track mitosis. As shown by Robinow and Caten (1969) bar-like masses of chromatin align on either side of a narrow spindle. According to Aist and Williams (1972) and Setliff et al. (1974) the

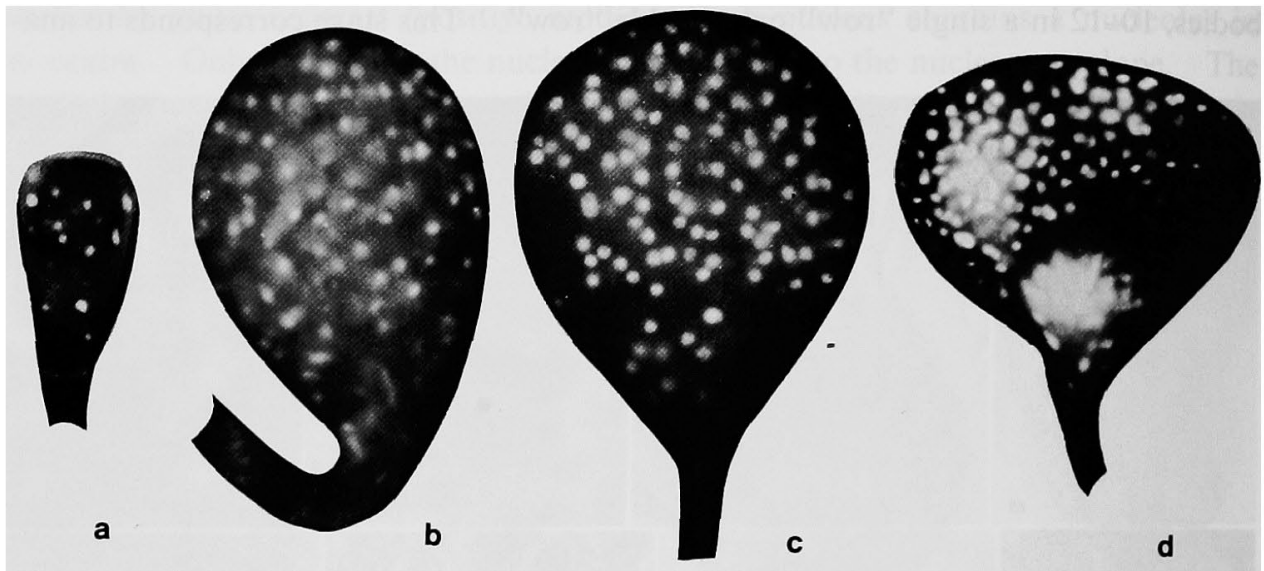

Fig. 4. (FFL) of development in vegetative sporangium of Absidia glauca. Single nuclei seen as fluorescing bright spots. b-d show stages during spore delimitation.
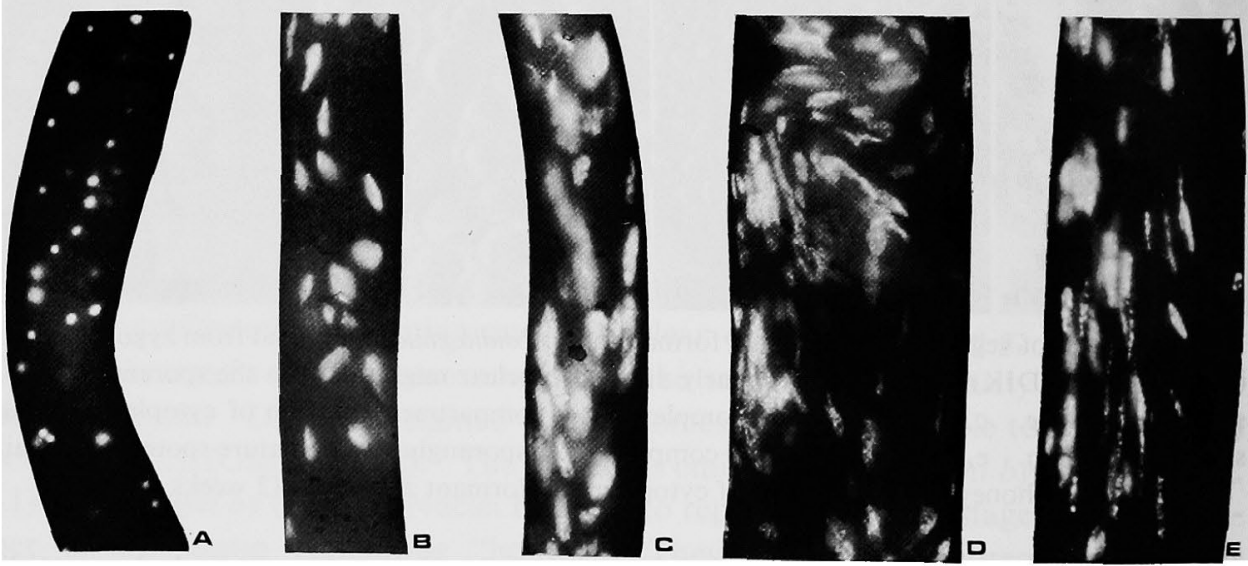

Fig. 5. (FFL) of hyphal nuclei in Absidia glauca. Nuclei are seen as bright spots. A, interphase. $\mathrm{B}$, early stages of nuclear division. C-E, later stages of nuclear division. Individual chromosomes and chromatids are distinct in $\mathrm{E}$.

two-track image arises because mitotic chromosomes or chromatids become arranged around the periphery of the central spindle, lying at various positions, staggered along the spindle length. Anaphase separation begins when the chromosomes are in such scattered array and a well defined metaphase plate is never formed. It is believed that optical sectioning of a more or less cylindrical chromatin distribution produces the two track images so often recorded. 
It appears to us, however, that a cylindrical chromatin distribution cannot be a sufficient explanation for the delicate two track pattern we observed with (FFL) in A. glauca in many cases. Improvements in fixation techniques, staining combined with serial sectioning and reconstruction analysis for (TEM) are necessary components to solve this problem. It is not yet clear whether chromosomal micro-

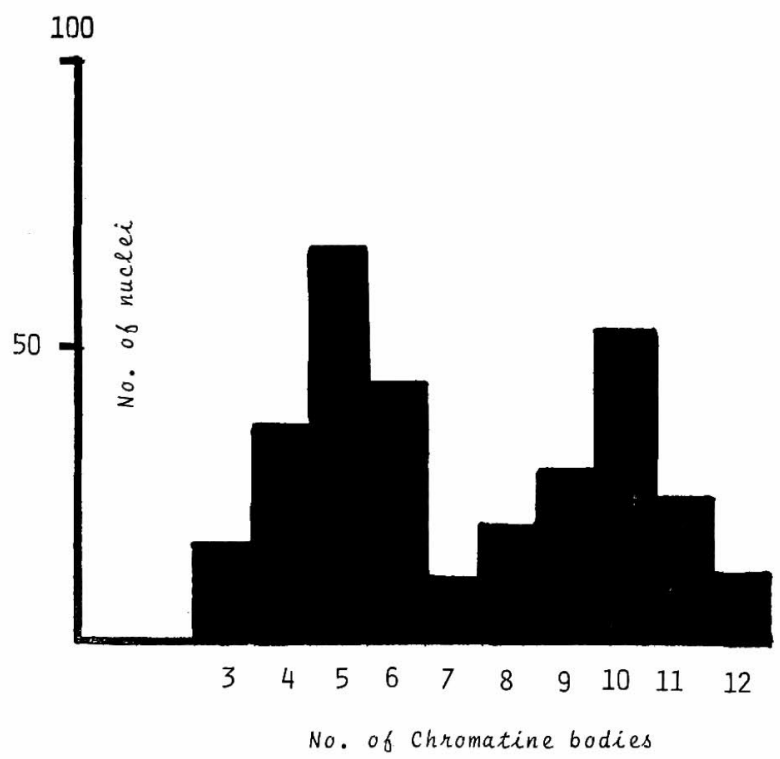

Fig. 6. Distribution of nuclear classes with different chromatin body numbers. The diagram is suggestive of a chromosome number of $n=5-6 . \quad 325$ nuclei analyzed.

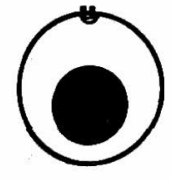

A

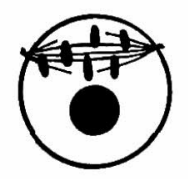

B

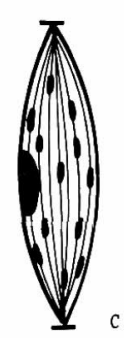

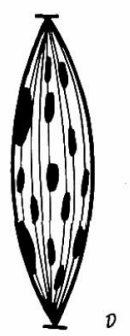

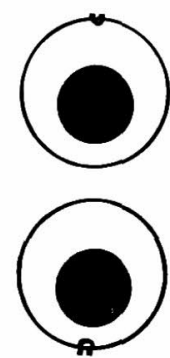

E

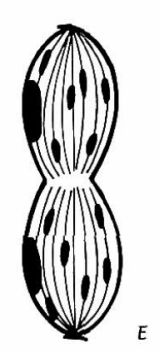

Fig. 7. Outline of vegetative nuclear division in Absidia glauca. Nuclear envelope is persistent, the spindle is organized by attached MTOCs, chromosome separation is asynchronous. B correspond to late prophase, C-E are anaphase-telophase. Precise details of chromosome arrangements are unknown.

tubules and kinetochores are commonly present among Zygomycetes (Heath 1978).

Vegetative spores

The vegetative spore swells during $2-4$ hours at $20^{\circ} \mathrm{C}$ (Fig. $1 \mathrm{a}-\mathrm{b}$ ). There is no dormant period. When the spore has reached about the double diameter (Fig. 
6), the single nucleus divides and 1-2 germination hyphae appear (Fig. $8 \mathrm{C}$ ). Rapid growth occurs with extensive mycelial branching. A coenocytic mycelium is produced.

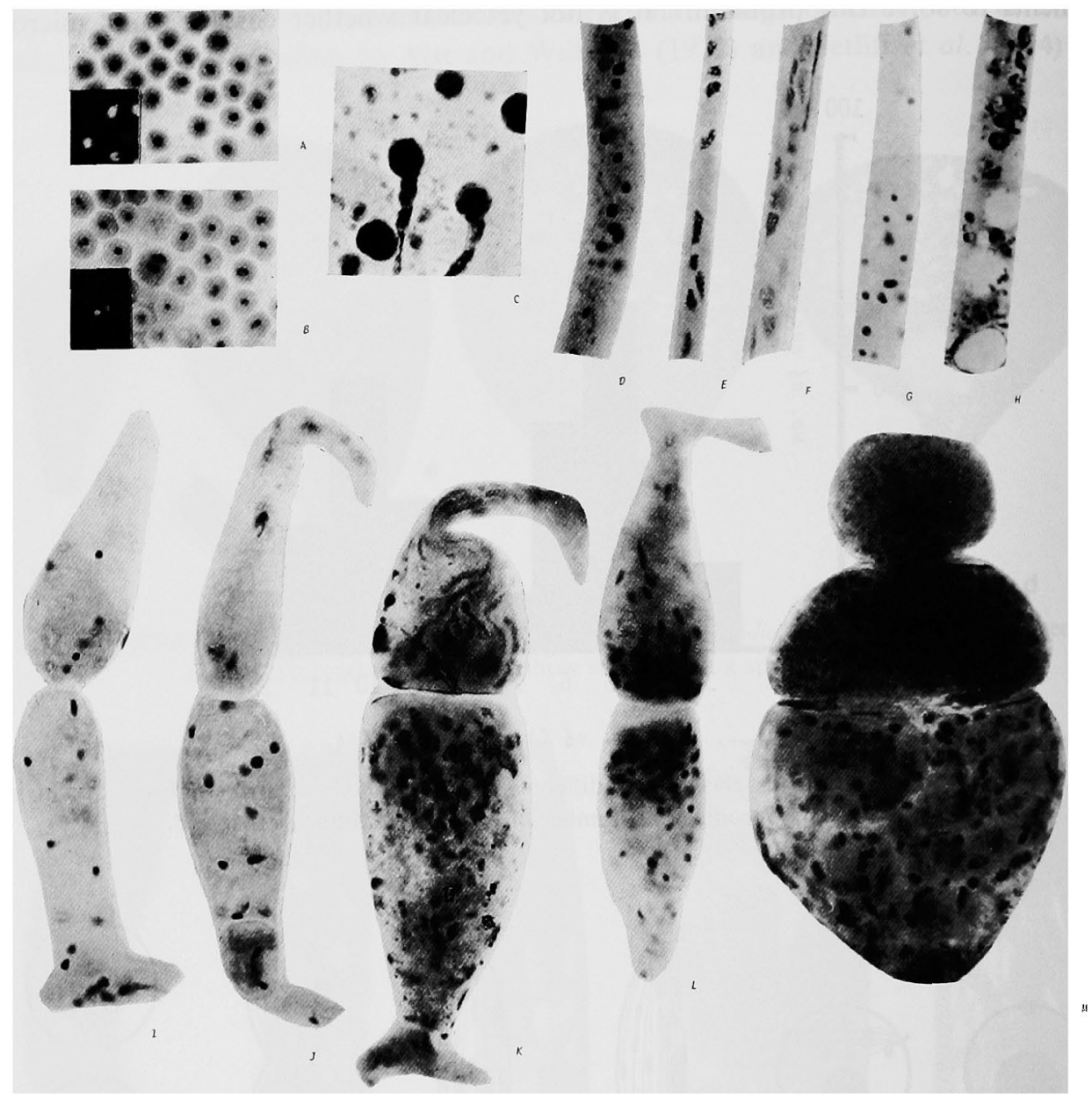

Fig. 8. Microphotographs, in part corresponding to drawings in Fig. 1. A, newly formed spores with a single nuclesus (OS) and (FFI)-insert. B, $4 \mathrm{~h}$ old swelled spores, showing primary division (OS) and (FFL)-insert. $\mathrm{C}, 6 \mathrm{~h}$ old spores with primary hypha. D-H, later stages showing multinucleate condition in hyphae, dividing nuclei are seen in E and F. I-M, development of the zygospore (OS). I-J, early stages, many more nuclei present than seen in focus. K, later stage, several nuclei have now migrated into the gametangia, note nuclear divisions in the $(-)$ gametangium. $\mathrm{L}$, aggregation of nuclei close to the fusion wall. M, later stage with partly fusing nuclei. (+)-gametangium contributes most to the zygospore. It contains in part fused nuclei, but also haploid ones. They are identified by size differences.

\section{Sporangium formation}

The vegetative sporangia form on special hyphae (Fig. 1, e-j, Fig. 2, a-e, Fig. 3,

Fig. 9. a-b, (TEM) of nuclei in 6 days old zygospores. Nucleus in $b$ shows early signs of degeneration (fibrillar area). $\mathrm{nc}=$ nucleolus. 


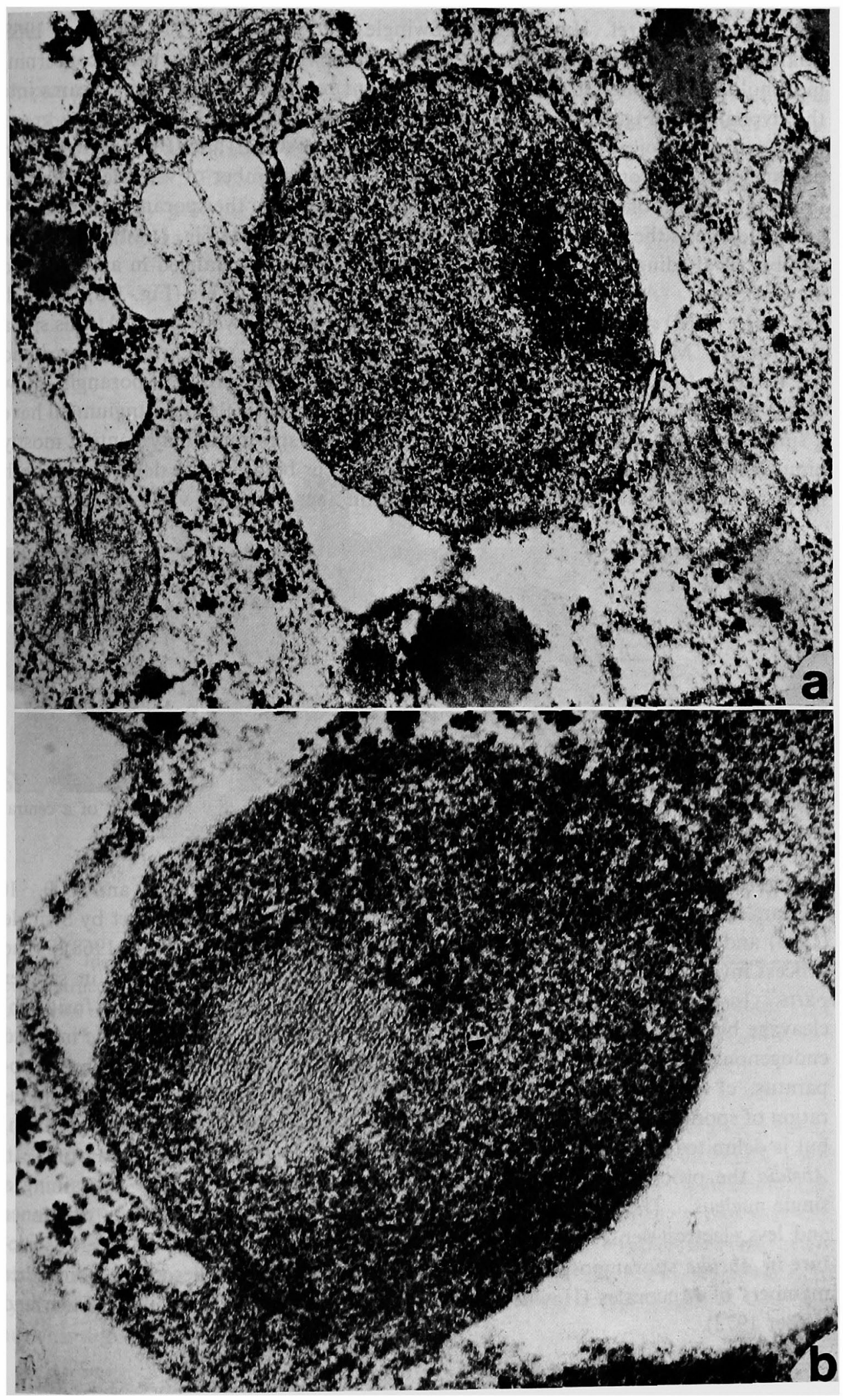


a-g, Fig. 4, a-d) (ref. Harper 1899, Swingle 1903, Moreau 1913, Bracker 1968, Laane 1974). Initially they appear not different from other hyphae concerning morphology and inner structure. Development begins as protoplasm streams into the hyphal end (Fig. 1, e-f, Fig. 2, A-C, Fig. 3, a-b, Fig. 4, a). The hypha grows negatively geotropically and swells in the tip. (OS), (AOFL) and (FFL) show numerous nuclei in division (Fig. $1 \mathrm{e}-\mathrm{g}$, Fig. 3, b). A large number of vacuoles is present in living preparations at this stage, being mostly located in the sporangial centre. A septum delimits the sporangium from the rest of the hyphae (Fig. 1, g-i). Now, the number of dividing nuclei decreases. The nuclei become arranged in an outer and an inner layer. A columella develops between these two layers (Fig. 1 h, Fig. $2 \mathrm{~d}$, Fig. 4 c). (LM) shows this to happen after fusion of small vacuoles. At this site a wall forms. Most nuclei in the columella disappear and a spore wall forms around the nuclei in the sporulation area (Fig. 3, d-e). Nearly all the sporangia show uninucleate spores and usually the mature spores in a particular sporangium all have a single nucleus (Fig. 2, e). However, $2-3 \%$ of the sporangia may contain mostly binucleate spores. Extensive nuclear divisions occur before spore delimitation both in the sporangium and columella. The final number of spores varies considerably.

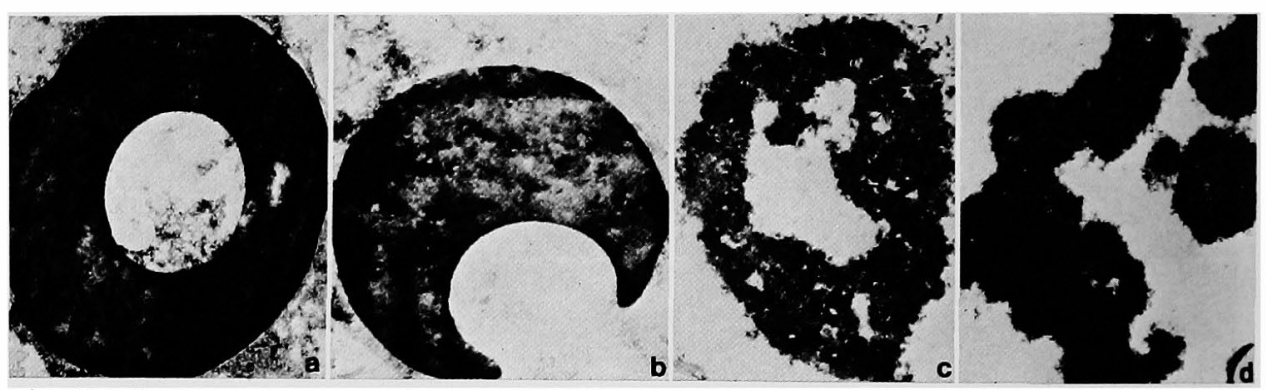

Fig. 10. Later stages of zygospore nuclear degeneration (a-d). Note development of a central vacuole, increasing osmiophily of nuclear material and gradual break-down (c-d).

The smallest sporangia have about 50 spores, the largest between 500 and 1000 . It appears that the spores are delimited by a process first observed in part by Swingle (1903) and later in detail with electron microscopy by Bracker $(1966,1968)$. The process involves furrowing by membrane material cutting the cytoplasm in smaller parts. In principle three stages occur; a) those prior to cleavage vesicle fusion, b) cleavage by vesicle fusion until spore protoplast delimitation with cleavage initiated endogenously by coalescence of cleavage vesicles to form a ramifying tubular apparatus, c) postcleavage which includes all further development leading to maturation of spores and columella. The columella is not an ordinary transverse septum, but is delimited by an apparatus structurally similar to the cleavage apparatus. In Absidia the process produces protoplasts of even size, each usually containing a single nucleus. The spore wall is thick, consisting of two major layers, one inner and less electron-dense, one outer and much more electrondense. The ultrastructure in Absidia sporangiospores appears rather similar to that described from other members of Mucorales (Hawker and Abbott 1963, Buckley, et al. 1968, Hess and Weber 1973). 


\section{The zygospore}

Zygospores form after $(+)$ and $(-)$ mycelia come in contact (Fig. 1, k, Fig. 8, i). A pair of opposed, aerial progametangia swell (Fig. 1, 1, Fig. 8, j) and the nuclear number in each increases due to cytoplasmatic migration and nuclear divisions. (LM) preparations stained with (OS) show nuclei in characteristic streaming arrangements (Fig. 8, k.). Each gametangium is delimited on either side of the fusion wall by centripetally growing gametangial septa (Fig. 1, 1). The progametangial part remaining after gametangial delimitaton, the socalled suspensor, contains a variable

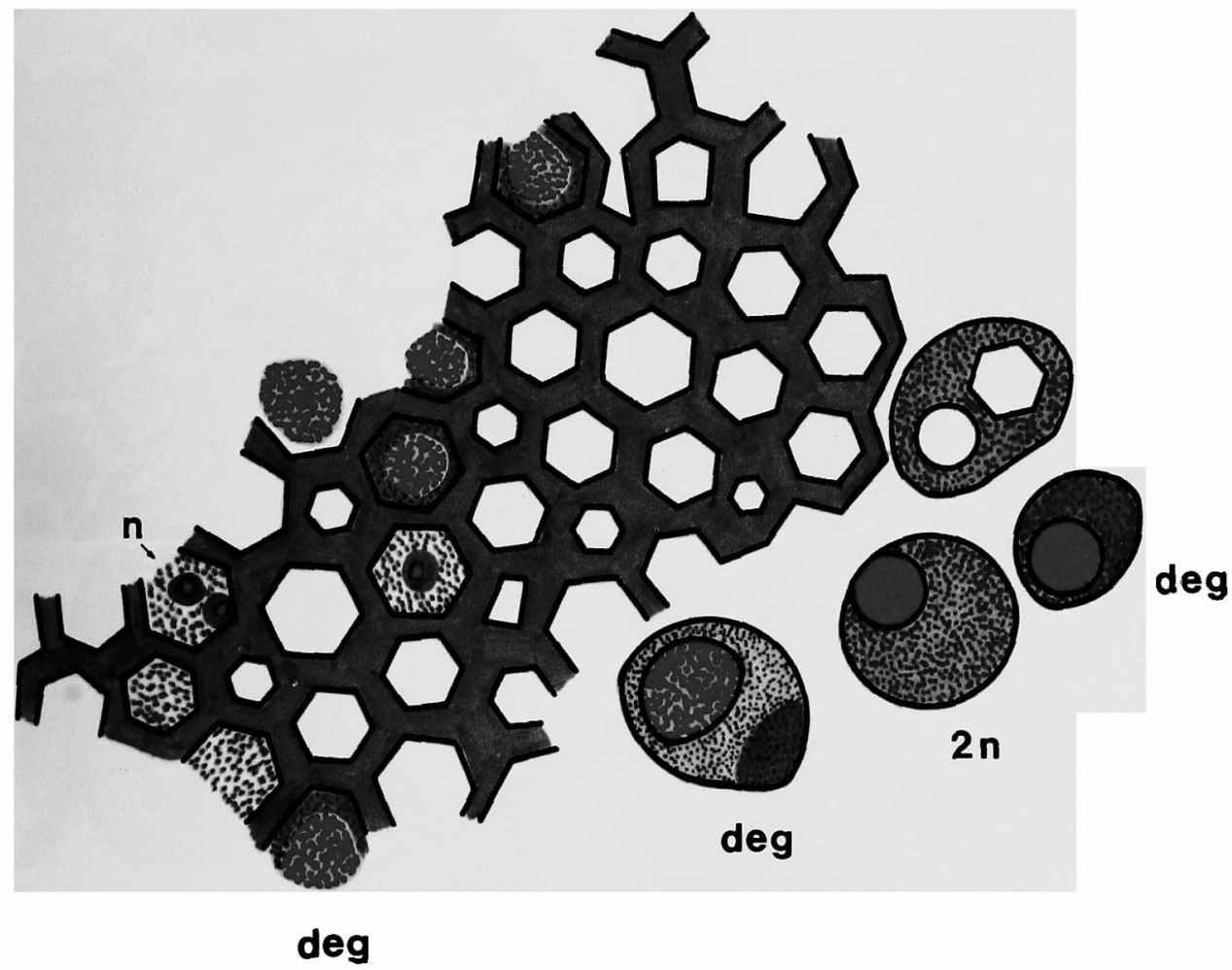

Fig. 11. Idealized drawing of content of 6 days old zygospore as seen by (AOFL). Note honeycomb structure of cytoplasm. Different nuclear types are seen, diploid ones marked $2 n$, degenerating ones marked deg and haploid ones marked $n$. Due to hypotonic swelling some nuclei have increased markedly in size. With this technique red fluorescence indiacte RNA, yellowgreen DNA.

number of nuclei which still may be dividing (Fig. 1, 1).

Upon completion of the gametangial septae the fusion wall undergoes a rapid dissolution process resulting in the fusion of the gametangial protoplasts. We counted the nuclei in various gametangia and found that $80 \%$ of them contained between 100-300 nuclei. At the stage of protoplast fusion (TEM) shows that the former "male" $(-)$ and "female" $(+)$ gametangium have a rather different cytoplasmatic structure with variable amounts of organelles. It is characteristic that zygospores older than 3-4 days become dark, loose their transparency, and the nuclei inside become difficult to examine. 


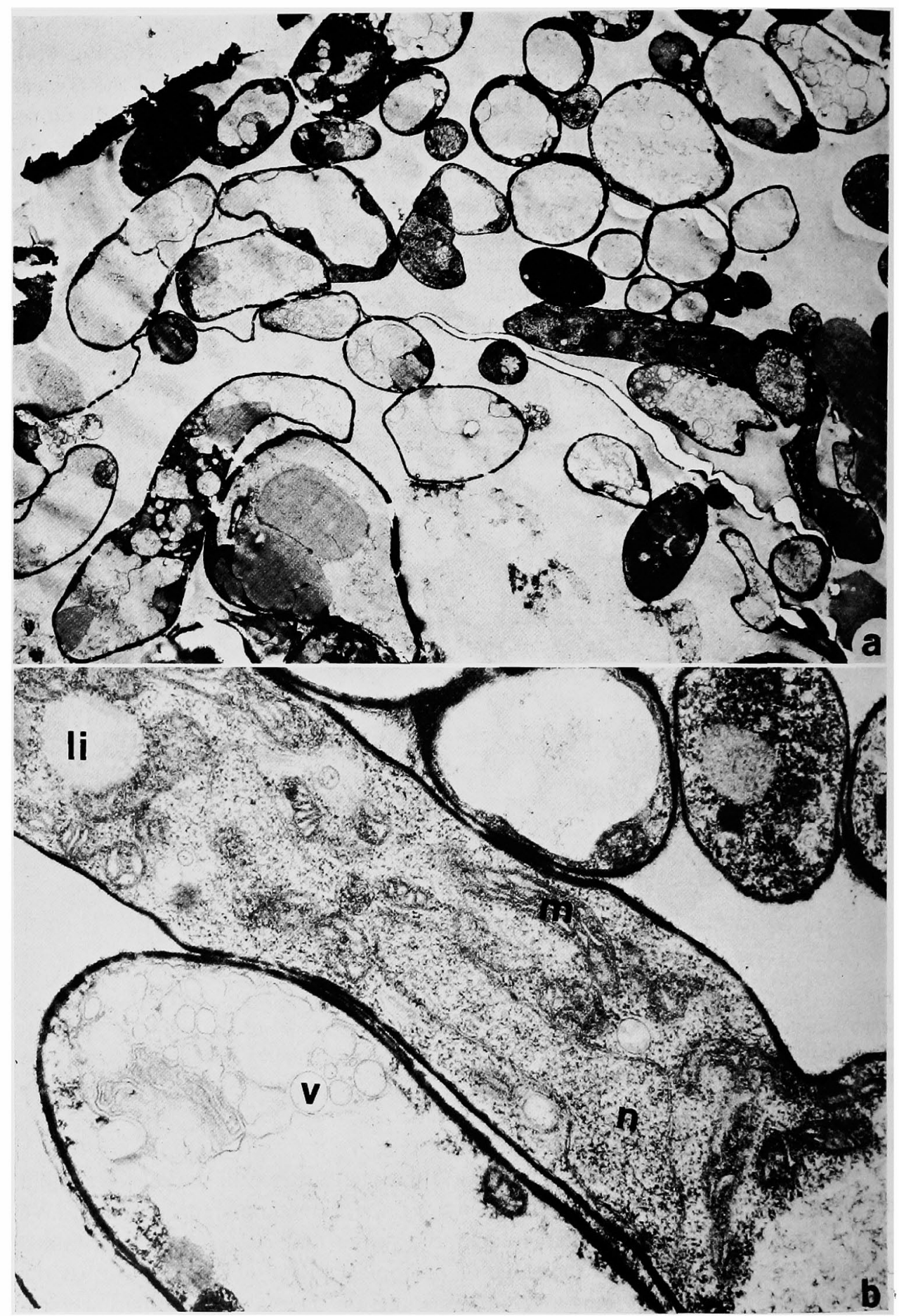

Fig. 12 a-b. (TEM) of section through germinating zygospore of Absidia glauca after 9 months dormancy. Hyphal material develops inside the zygospore. $\quad \mathrm{l}=$ lipid body, $\mathrm{m}=$ mitochondrium, $\mathrm{n}=$ nucleus, $\mathrm{v}=$ vacuole. 
We found the following.

(A) The nuclei inside the forming spore are of even size just after gametangial fusion. They are probably haploid (n).

(B) When the fusion wall had mostly disappeared, the even sized nuclei become arranged in pairs (Fig. 1m, Fig. $8 \mathrm{~m}$ ).

(C) In this and later stages different nuclear classes appear. Large nuclei with weak staining behaviour are diploid fusion nuclei $(2 n)$. Different sized, denser staining nuclei $(2 n$ and $n$ ) are in the process of nuclear degeneration (Fig. $9 a-b$, Fig. 11). Small nuclei with weak staining behaviour are unfused haploid ones (n) (Fig. 1n, 8m, 11).

(D) It seems that a few diploid and haploid nuclei are present both just before dormancy and later (Fig. 11). The cytoplasm in such spores forms often a honeycomb like pattern with the few remaining nuclei situated in the holes. Other holes contain socalled "oil-plastids" (Cutter 1942a, b).

Cutter (1942a) found in 6-7 days old zygospores of $A$. spinosa division figures that appeared to be meiotic. These, probably meiotic nuclei, were many times the size of the usual vegetative nuclei and may well correspond to the nucleus drawn in Fig. 11 marked 2n. According to Cutter his observations gave added credence to Bairds (1924) contention that the socalled oil-plastids arise within the nuclei (see our Fig. 9). Cutter was unable to observe stages later than diakinesis in this species. Shortly later, about twice the number of apparently haploid nuclei appeared. In A. glauca (Cutter 1942b) also the fusion nuclei were prominent and all supernumerary nuclei appeared to degenerate before the zygospore becomes dormant. Due to the great abundance of oil plastids it was not possible to ascertain whether all nuclei remained in fused condition and no evidence of nuclear division of any sort was seen between onset of dormancy and germination.

\section{Zygospore germination}

In classic descriptions of Zygomycetes the zygospore is described to germinate and form a special kind of sporangium, the socalled germ sporangium (Fig. 13). Germ sporangia have, however, only been observed a few times in A. glauca, more frequently in A. spinosa (Blakeslee 1906, Cutter 1942b). Germination may occur 6-7 months after onset of dormancy. Sometimes heat-treatment (Halbsguth and Rudolph 1962), $\mathrm{NH}_{4}$-acetate or acetic acid (Delwaux 1973) may break the dormancy, but we found such treatment not to work in our case. None of the several thousand examined zygospores developed germ sporangia. We found, however, that extensive hyphal masses (Fig. 12) developed inside intact zygospores that had been carefully sterilized by hypochlorite. We exclude the possibility of contamination from adhering, surviving and germinating vegetative spores. Hyphal development occurred in zygospores up to three months old. It appears that $A$. glauca has extensive possibilities of forming mycelia inside zygospores which finally burst and form a vegetative mycelium. We have thus included this possibility in the scheme of life-cyccle in A. glauca (Fig. 13). A report by Hocking (1967) from studies of Phycomyces blakesleeanus shows that at least immature zygospores could be induced to undergo precocious germination with hyphal growth. These germinating zygospo- 
res were, however, young, --only 2-4 days old and had not yet developed the thick, dark exospore.

The nuclear behaviour during such zygospore mycelial formation is unknown and technical improvements are necessary in order to perform a cytological analy-

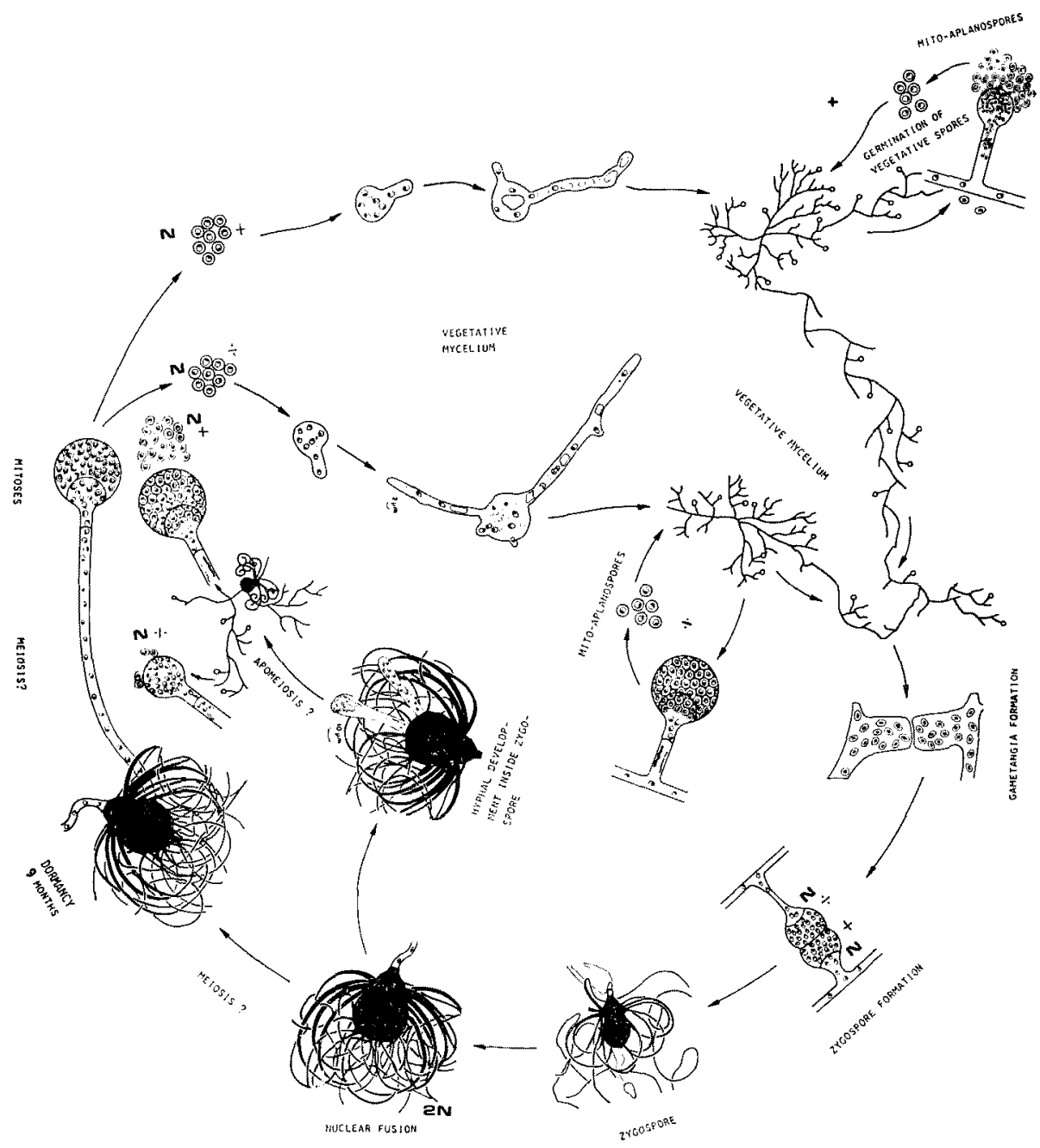

Fig. 13. Summarized diagram of life-cycle in Absidia glauca. (+) and (-) strains may reproduce vegetatively, but may form zygospores when coming in contact. After nuclear fusion in the zygospores the spores contain diploid and haploid nuclei, the latter of different mating types. Both a number of diploid and haploid nuclei degenerate. After a dormant period, a germ sporangium may form, but more usual hyphal growth occurs inside the sporangium and vegetative spores are formed. By this mode it is suggested that diploid nuclei may also become haploid by successive chromosome loss. Existence and timing of ordinary meiosis are unknown.

sis. The possibility of exclusion of meiosis in favour of successive chromosome loss from remaining diploid nuclei might be considered. 


\section{Summary}

1. The nuclear behaviour and life-cycle in Absidia glauca (Mucorales) have been examined by a variety of microscopical techniques.

2. The uninucleate sporangiospores germinate to produce a coenocytic multinucleate mycelium. Vegetative nuclear division involves persistent nuclear envelope, asynchronous chromatid separation and lack of a metaphase plate. Sporangiospores formed inside the sporangium by a process of progressive cleavage, each protoplast containing a single nucleus.

3. Zygospores form after fusion by $(+)$ and $(-)$ gametangia on different mycelia. Nuclei fuse in pairs in the forming zygospore, but some nuclei remain haploid. Before zygospore dormancy a number of both haploid and diploid fusion nuclei degenerate. Remaining fusion nuclei are enlarged and probably in meiotic prophase before dormancy. Meiotic stages were not observed.

4. Zygospores did not germinate after dormancy by forming germ sporangia. Instead, hyphal development occurred inside a number of zygospores and a vegetative mycelium was produced. It is not known whether diploid nuclei divide meiotically or become haploid by successive chromosome loss during this process.

\section{References}

Aist, J.R. and Williams, P. H. 1972. Ultrastructure and time course of mitosis in the fungus Fusarium oxysporum. J. Cell Biol. 5 : 368-398.

Baird, E. A. 1924. The structure and behaviour of the nucleus in the life history of Phycomyces nitens (Agardh) Kunze and Rhizopus nigricans Ehrbg. Trans. Wisconsin Acad. 21 : 357380.

Blakeslee, A. F. 1904. Sexual reproduction in the Mucorineae. Proc. Amer. Acad. Arts and Sci. 40: 205-319.

- 1906. Zygospore germination in the Mucorineae. Annales Mycologici 4: 1-28.

Bracker, C. E. 1966. Ultrastructural aspects of sporangiospore formation in Gilbertella persicaria. In "The Fungus Spore", M. F. Madelin (Ed.). Proc. 18th Symp. Colston Res. Soc. Butterworth Sci. Publ. London. pp. 39-60.

- 1968. The ultrastructure and development of sporangia in Gilbertella persicaria. Mycologia 60: $1016-1067$.

Buckley, P. M., Commer, N. F. and Matsumoto, T. T. 1968. Ultrastructural details in germinating sporangiospores of Rhizopus stolonifer and Rhizopus arrhizus. J. Bacteriol. 95: 23652373.

Cerda-Olmeido, E. 1975. The genetics of Phycomyces blakesleeanus. Genet. Res. Cambr. 25: 285-296.

Cutter, V. M. 1942a. Nuclear behaviour in the Mucorales. I. The Mucor pattern. Bull. Torrey Bot. Club 69: 480-508.

- 1942b. Nuclear behaviour in the Mucorales. II. The Rhizopus, Phycomyces and Sporodinia patterns. Bull. Torrey Bot. Club 69 : 592-616.

Dangeard, P. A. and Leger, M. 1894a. Recherches sur la structure des Mucorinées. Botaniste 4: 4-11.

- and - 1894b. Recherches sur la structure des Mucorinées. Comt. Rend. Acad. Sci. Paris 18: $430-432$.

Day, A. W. 1972. Genetic implications of current models of somatic nuclear division on fungi.

Can. J. Bot. 50: 1337-1347.

Delwaux, E. 1973. Some aspects of germination induction in Phycomyces blakesleeanus by an 
ammonium acetate pretreatment. Arch. Microbiol. 88: 273-284.

Ehrenberg, C.F. 1820. Syzygites, eine neue Schimmelpilzgattung nebst Beobachtungen über sichtbare Bewegung in Schimmeln. Verh. Gesellsch. Nat. Freund. Berlin 1.

Eslava, A. P., Alvarez, M. I., Burke, P. V. and Delbrück, M. 1975. Genetic recombination in sexual crosses of Phycomyces. Genetics 80: 445-462.

Esser, K. 1982. Cryptogams. Cyanobacteria, Algae, Fungi, Lichens. Cambridge University Press, Cambridge.

Franke, W. W. and Reau, P. 1972. The mitotic apparatus for a zygomycete, Phycomyces blakesleeanus. Arch. Microbiol. 90: 121-129.

Gauger, W. L. 1977. Meiotic gene segregation in Rhizopus stolonifer. J. Gen. Microbiol. 101: 211-217.

Hagem, O. 1908. Untersuchungen über norwegische Mucorineen. Skr. Vidensk. Selsk. Christ. I. Mat. Nat. K1. No. 7: 1-50.

Halbsguth, W. and Rudolph, H. 1962. Untersuchungen über die Wärmeaktivierung der Sporangio* sporen von Phycomyces blakesleeanus. Beitr. Biol. Pflanzen 38: 31-61.

Harper, R. A. 1899. Cell division in sporangia and asci. Ann. Bot. 13: 467-515.

Hawker, L. E. and Abbott, P. V. 1963. An electron microscope study of maturation and germination of sporangiospores of two species of Rihzopus. J. Gen. Microbiol. 32: 295-298.

Heath, I. B. 1978. Nuclear Division in the Fungi. Academic Press, New York.

Hesss, W. M. and Weber, D. J. 1973. Ultrastructure of dormant and germinated sporangiospores of Rhizopus arrhizus. Protoplasma 77: 15-33.

Hocking, D. 1967. Zygospore initiation, development and germination in Phycomyces blakesleeanus. Trans. Br. Mycol. Soc. 50: 975-980.

Keene, M. L. 1914. Cytological studies of the zygospore of Sporodinia grandis. Ann. Bot. 28: 455-470.

Kubai, D. 1978. "Mitosis and Fungal Phylogeny". In Heath, I. B. (Ed.) Nuclear Division in the Fungi. Academic Press, New York, pp. 177-229.

Laane, M. M. 1974. Nuclear behaviour during the vegetative stage and zygospore formation in Absidia glauca. Norw. J. Bot. 21 : 125-135.

- and Lie, T. 1975. Exanimation of fungal nuclei with the Feulgen fluorescence method. Mikroskopie 31: 85-90.

Lendner, A. 1908. Les Mucorniées de la Suisse. Berne. pp. 1-180.

Ling-Young, M. 1930. Études Biologique des phénomènes de la sexualité chez les Mucorinées. Rev. Gén. Bot. 42: 618-639.

Moreau, F. 1913. Recherches sur la reproduction des Mucorinées. Botaniste 13: 1-136.

Olson, L. W. 1974. Meiosis in the aquatic phycomycete Allomyces macrogynus. C. R. Trav. Lab. Carlsb. 40: 114-124.

Robinow, C. F. and Caten, C. E. 1969. Mitosis in Aspergillus nidulans. J. Cell Sci. 5: 403-431.

Setliff, E. C., Hoch, H. C. and Patton, R. F. 1974. Studies on nuclear division on the basidia of Poria latemarginata. Ca. J. Bot. 52: 2323-2334.

Swingle, D. B. 1903. Formation of the spores of Rhizopus nigricans and Phycomyces nitens. Bull. U. S. Bur. Plant. Ind. 37: 1-40. 